\title{
FUNCIONAMIENTO FAMILIAR Y TRASTORNOS DE LA CONDUCTA ALIMENTARIA. UNA INVESTIGACIÓN DESDE EL MODELO CIRCUMPLEJO
}

\author{
FAMILY FUNCTIONING AND EATING DISORDERS. A PERSPECTIVE FROM THE \\ CIRCUMPLEX MODEL
}

\author{
Milagros Fernández Ruiz \\ Nuria Masjuan \\ Daniel Costa-Ball \\ Cecilia Cracco
}

Facultad de Psicología, Universidad Católica del Uruguay

\begin{abstract}
Resumen: El objetivo de este trabajo ha sido describir el funcionamiento de las familias con un miembro con Trastorno de la Conducta Alimentaria (TCA) y comparar su funcionamiento con familias normativas según las variables del Modelo Circumplejo. La muestra estuvo conformada por 63 participantes, miembros de 16 familias uruguayas en las que uno de sus miembros se encontraba en tratamiento de rehabilitación específico. Los resultados indicaron que estas familias poseen poca cohesión y flexibilidad en comparación con las familias normativas, mostrándose en su gran mayoría con un funcionamiento caótico, desligado, enmarañado y rígido con mayores dificultades en la comunicación y con baja satisfacción familiar. Considerando el rol, se encontraron diferencias en la percepción del funcionamiento familiar principalmente entre los padres y el resto de los miembros de las familias. Estos resultados plantean a los clínicos la necesidad de trabajar con todo el sistema familiar para abordar la problemática de los TCA.
\end{abstract}

\section{Palabras Clave: Funcionamiento Familiar,;Trastornos Alimentarios; Modelo Circumplejo}

\begin{abstract}
The aim of this study was to assess the family functioning of those with a member diagnosed with eating Disorder (ED) and to compare with normative families according with the circumflex model. Sixteen Uruguayan families with a member in rehabilitation were assessed. Results suggest that these families showed in terms of the circumflex model less cohesion and flexibility, and a chaotic, disengaged, enmeshed, rigid functioning with difficulties in communication and less family satisfaction, compared with the normative families. Differences in parents and the rest of the family member perceptions of the family functioning have been found considering the role. These results present to the clinicians the need to work with all the family system in order to approach the ED.
\end{abstract}

Key Words: Family Functioning; Eating Disorders; Circumplex Model

\section{Introducción}

Los Trastornos de la Conducta Alimentaria (TCA) son patologías altamente frecuentes en la población adolescente, con repercusiones graves en la salud y dificultades en su abordaje terapéutico (Correa, Zubarew, Silva \& Romero, 2006; Cruzat, Ramírez, Melipillán \& Marzolo, 2008). Entre los trastornos más conocidos se destacan la Anorexia Nerviosa y la Bulimia Nerviosa. La prevalencia de la Anorexia Nerviosa es entre $0.5 \%$ y $1 \%$, y el de la Bulimia Nerviosa es entre 1 y 3\%, según el DSM 5 (APA, 2013).

Diferentes escuelas sistémicas ofrecen explicaciones sobre los TCA y el funcionamiento familiar. Espina (1996) destaca tres modelos: el Modelo Estructural que describe a la familia de las anoréxicas como psicosomáticas identificando como características principales de estas familias el enmarañamiento, la sobreprotección, la rigidez, la evitación y baja resolución de los conflictos; el Modelo Comunicacional que sostiene que las familias con hijas con anorexia tienen problemas en la comunicación, dificultades de liderazgo en el sistema parental, alianzas y coaliciones negadas; y el Modelo Transgeneracional que plantea que la familia de la anoréxica funciona como un sistema rígido de creencias implícitas que se transmiten de generación en generación con la existencia de dificultades para la individuación de sus miembros y la solución de problemas.

Holtom-Viesel y Allan (2014) señalan que varias investigaciones sobre el funcionamiento 
familiar han sido criticados por asumir causalidad, culpabilizando a la familia. Recientemente, los estudios se han centrado en la familia como factor de mantenimiento en los TCA, destacando su rol en los procesos de recuperación.

Uno de los modelos particularmente útil para el diagnóstico relacional de las familias con TCA y que ha sido utilizado por varios investigadores (Cook-Darzens, Doyen, Falissard \& Mouren, 2005; Manglio \& Molina, 2012; March et al., 2006; Ruíz, Vazquez, Mancilla, Viladrich \& Castillo, 2013; Vidovic, Jures, Begovac, Mahnik \& Tocilj, 2005), es el Modelo Circumplejo de los Sistemas Maritales y Familiares, desarrollado por Olson, Sprenkle y Russell (1979). Este modelo integra tres dimensiones: cohesión, flexibilidad y comunicación (Olson, 2011), que se han encontrado como relevantes en diversas teorías y abordajes clínicos (Olson, 2000).

La dimensión cohesión es definida como los lazos familiares que los miembros de la familia tienen entre sí. Es una medida del grado de proximidad o separación respecto al tiempo compartido, amigos, espacio, toma de decisiones, intereses y recreación. Se consideran cinco niveles de cohesión: desligado, separado, conectado, muy conectado y enmarañado (Olson, 2000; 2011). Los dos niveles que se encuentran en los extremos (desligado - enmarañado), reflejan un funcionamiento familiar problemático, mientras que los tres niveles centrales restantes (separado, conectado y muy conectado) se asocian a un óptimo funcionamiento familiar (Olson \& Gorall, 2003). En uno de los extremos se encuentran las familias enmarañadas, las cuales dificultan la individuación de sus integrantes. En estas familias, los individuos son muy dependientes, existiendo poco espacio para la privacidad, demandando mucha lealtad a la familia y focalizando poco en intereses fuera de la familia. En el otro extremo, se encuentran las familias desligadas, las cuales estimulan en gran medida el desarrollo de la autonomía, pero no promueven sentimientos de seguridad y pertenencia (Olson \& Gorall, 2003).

La dimensión flexibilidad es definida como la capacidad de cambio en el liderazgo familiar, los roles y las reglas del sistema. Se consideran cinco niveles de flexibilidad: rígido, poco flexible, flexible, muy flexible y caótico. La hipótesis que maneja Olson (2011), es que los niveles balanceados de flexibilidad (poco flexible, flexible y muy flexible) son más funcionales que los extremos (rígidos o caóticos). Esto se debería a que las familias necesitan tanto de la estabilidad como de la capacidad de cambio para poder ser funcionales.

La comunicación es considerada como una dimensión facilitadora para permitir el movimiento en las otras dos dimensiones: cohesión y flexibilidad. La dimensión comunicación es definida por las habilidades de comunicación positiva (mensajes claros y congruentes, empatía, frases de apoyo y habilidades efectivas para la resolución de problemas). Varios estudios que han investigado la comunicación y la capacidad para resolver problemas en parejas y familias, encontraron que los sistemas balanceados en cohesión y flexibilidad tienden a tener mejor comunicación, mientras que sistemas desbalanceados en estas dos dimensiones tienden a una pobre comunicación (Olson \& Gorall, 2003).

Para la evaluación de las tres dimensiones del modelo circumplejo fue creado el instrumento FACES en el año 1978, con modificaciones en el FACES // en 1982, FACES III en 1985 y por último el FACES IV en 1996 (Olson, 2000). Este instrumento está diseñado para evaluar la cohesión, flexibilidad y comunicación familiar según el Modelo Circumplejo de los Sistemas Maritales y es uno de los métodos de evaluación familiar más utilizados para evaluar la cohesión familiar y la flexibilidad familiar. Las hipótesis básicas relacionadas con el Modelo Circumplejo son los siguientes: niveles balanceados de cohesión y flexibilidad conducen a un funcionamiento familiar saludable; niveles desbalanceados de cohesión y flexibilidad se asocian con un funcionamiento familiar problemático (Olson, 2000). Una revisión realizada por Kouneski (2000) mostró que distintas versiones del FACES han sido utilizadas en más de 1.200 artículos en todo el mundo.

Si bien no forma parte del Modelo Circumplejo, la satisfacción familiar, es una variable que ha sido ampliamente estudiada en investigaciones en las que se tomó este modelo, como una forma posible de validar sus hipótesis básicas (Olson \& Gorall, 2003). La Satisfacción Familiar se define como el grado de complacencia de los miembros de la familia respecto a las relaciones que establecen entre si (Olson, 2011). 
Si bien parece no haber un patrón específico de funcionamiento familiar asociado a los TCA, estas familias tienden a ser más disfuncionales que las familias control (Cruzat, Ramírez, Melipillán \& Marzolo, 2008; Ruiz, Vázquez, Mancilla, Viladrich \& Castillo, 2013), mostrando menor cohesión (Dancyger, Fornari, Scionti, Wistsky \& Sunday, 2005; Manglio \& Molina, 2012; Vázquez, Ruíz, Álvarez, Mancilla \& Tena, 2010), mayor rigidez (Holtom-Viesel \& Allan, 2014), falta de flexibilidad (Vidovic et al., 2005) y dificultades en la comunicación (Crispo, Figueroa \& Guelar, 1996; Selvini-Palazzoli, Cirillo, Selvini \& Sorrentino, 1999).

En 2014, Holtom-Viesel y Allan realizaron una revisión sistemática de las investigaciones que compararon el funcionamiento familiar de familias con TCA y familias control. Los criterios de inclusión en la búsqueda de investigaciones fueron: uso de metodología cuantitativa, foco en el funcionamiento familiar y TCA; y que los participantes fueran diagnosticados con TCA siguiendo los criterios del DSM o ICD. Los autores seleccionaron 17 investigaciones de un total de 1156 que fueron analizadas en función de los criterios especificados. Dejaron fuera los estudios que consideraban al funcionamiento familiar como factor etiológico de los TCA. Del análisis de las investigaciones seleccionadas, se desprende que existe un peor funcionamiento familiar en familias con TCA en comparación con las familias control; no se puede afirmar que exista un estilo disfuncional propio de las familias con TCA; los pacientes con TCA reportan mayor disfuncionalidad familiar que los padres; y los pacientes con percepciones más positivas de funcionamiento familiar obtienen una mejor recuperación. Los autores señalan algunas limitaciones de estas conclusiones debido a lo inconsistente de los resultados y cuestiones de orden metodológico.

En Uruguay, Feibuscheurez y Masjuan (2009) realizaron un estudio intentando aproximarse al funcionamiento de una familia con un miembro con Anorexia Nerviosa (AN) y al de una familia con un miembro con Bulimia Nerviosa (BN) utilizando la escala FACES IV (2011). Los resultados mostraron que a nivel global, las dos familias reportaron un funcionamiento más adecuado en contraste a la percepción de las pacientes identificadas, que mostraron una visión diferente y menos positiva del funcionamiento familiar. Los resultados de este trabajo realizado en Uruguay, están en la misma línea de otras investigaciones que evidencian que existen discrepancias en la percepción del funcionamiento dependiendo del rol que cada miembro desempeña (Dancyger et al 2005; Ruiz et al, 2013; Holtom-Viesel \& Allan, 2014).

En relación al rol, Manglio y Molina (2012) reportaron que en las familias con TCA existe acuerdo entre las adolescentes con TCA y sus hermanos en describir un patrón comunicacional más negativo. En la misma línea Vidovic et al. (2005) reportaron que las hijas tienden a ser más críticas del funcionamiento familiar que las madres. Dancyger et al. (2005) también encontraron diferencias en la percepción del funcionamiento familiar, las hijas y los padres reportaron un funcionamiento más disfuncional en comparación a las madres. Las discrepancias son amplias, en algunos estudios se encuentran similitudes entre hijas y madres y discrepancias con el padre y otros, las diferencias ocurren entre padre y madre (Ruiz et al., 2007).

Estos resultados contradictorios en relación al funcionamiento familiar, junto a la ausencia de estudios en nuestro país, nos llevó a continuar profundizando en la comprensión del funcionamiento familiar de los trastornos Alimentarios en Uruguay. Por tal motivo, se realizó un estudio empírico con los siguientes objetivos: describir el funcionamiento de una pequeña muestra de familias con un miembro adolescente con TCA en Uruguay, según las variables del Modelo Circumplejo, comparando el funcionamiento familiar de las familias con un miembro adolescente con TCA, con el funcionamiento familiar de familias normativas con hijos en etapa adolescente.

\section{Método}

\section{Participantes}

La muestra estuvo conformada por 16 familias con un integrante con diagnóstico de Trastorno alimentario específico según los criterios del DSM-IV que se encontraban en tratamiento de rehabilitación en un centro privado de atención a los trastornos alimentarios de Montevideo (Uruguay). El diagnóstico de TCA fue efectuado por el equipo terapéutico de la clínica tratante. Cada grupo familiar estuvo conformado por la madre, padre, un integrante con diagnóstico de TCA y el hermano más cercano en edad con el paciente, 
según la metodología propuesta por Manglio y Molina (2012).

Todas las familias pertenecieron a un nivel socioeconómico medio y medio alto. La muestra quedó conformada por 63 participantes, 16 pacientes con TCA, 15 padres, 16 madres y 16 hermanos. La edad media de las pacientes con TCA fue de 17.06 ( $D E=2.62)$. Las madres presentaron una edad media $=46.25(D E=$ $4,34)$, los padres una edad media $=50.07$ $(D E=6,10)$ y los hermanos una edad media $=$ $19,18(D E=3,62)$.

El sexo de las pacientes es femenino y las mismas se encontraban en tratamiento ambulatorio con una modalidad de Hospital de día, cursando el primer año de tratamiento.Todas residían en la ciudad de Montevideo.

\section{Instrumentos}

- Escala de Evaluación de la Adaptación y Cohesión familiar (FACES IV; Olson, 2011) en su versión abreviada y adaptada al Uruguay por Costa-Ball, González Tornaría, del Arca, Masjuan y Olson (2013). El paquete de FACES IV sugiere sea completado por todos los miembros del hogar de 12 años o más edad. FACES IV utiliza preguntas con formato de escala Likert para ayudar a identificar la dinámica familiar. Esta versión cuenta con 24 ítems divididos en dos escalas balanceadas (cohesión y flexibilidad) y cuatro escalas desbalanceadas que miden los extremos inferior y superior de la primera dimensión cohesión (enmarañada y desligada) y de la segunda dimensión flexibilidad (caos y rigidez). Cada una de estas escalas está conformada por cuatro ítems y todas ellas se responden con una escala tipo likert de cinco opciones. Se realizó un análisis factorial confirmatorio con todos los ítems del FACES IV, encontrándose un ajuste adecuado del modelo de seis factores: dos factores balanceados (cohesión y flexibilidad) y cuatro desbalanceados (desligado, enmarañado, rígido y caótico). Las escalas probaron ser válidas y con valores de fiabilidad adecuados para trabajar en investigación (Costa Ball, et al., 2013).

- FCS (Family Communication Scale): fue diseñada por Barnes y Olson en 1982, para evaluar la tercera dimensión del Modelo Circunflejo. La comunicación es considerada como una dimensión facilitadora que ayuda a las familias moverse en las otras dos dimensiones del modelo (cohesión y flexibilidad). Evalúa las habilidades positivas de comunicación en una familia: mensajes claros y congruentes, empatía, apoyo, resolución de problemas, confianza y tono emocional de las interacciones. La puntuación total se obtiene de la sumatoria de las puntuaciones otorgadas en cada uno de los ítems. La puntuación mínima que se puede obtener con la escala es de 10 puntos y la puntación máxima es de 50 puntos. Las puntaciones más altas indican una mejor comunicación familiar (Sanz Vázquez, 2008a). Se utilizó la versión adaptada al Uruguay por Costa Ball et al. (2009). Esta escala está conformada por 10 ítems que se responden con una escala tipo likert de cinco opciones. Presentó una fiabilidad adecuada (.88) en la versión uruguaya (Costa Ball et al., 2009).

- FSS (Family Satisfaction Scale): Fue diseñada por Olson y Wilson en 1982, evalúa el grado de satisfacción que experimenta una familia sobre su nivel de cohesión y flexibilidad familiar, en relación a diez aspectos de la dinámica familiar, cinco relacionados con la cohesión y el resto con la flexibilidad familiar. Esta escala fue desarrollada con el objetivo de evaluar el grado de satisfacción que experimenta una familia sobre su nivel de cohesión y flexibilidad en relación al Modelo Circumplejo (Sanz Vázquez, 2008b). La puntuación total se obtiene de la sumatoria de las puntuaciones otorgadas en cada uno de los ítems. La puntuación mínima que se puede obtener con la escala es de 10 puntos y la puntación máxima es de 50 puntos. Las puntaciones más altas indican una mejor comunicación familiar. Se utilizó la versión adaptada al Uruguay por Costa-Ball et al. (2013). Esta escala está conformada por 10 ítems que se responden con una escala tipo likert de cinco opciones. Presentó una alta fiabilidad (.94) en la versión uruguaya (Costa-Ball et al., 2009).

\section{Procedimiento}

Se concurrió a una clínica especializada en los Trastornos Alimentarios privada de referencia en Montevideo y se mantuvo una entrevista con la coordinadora del equipo terapéutico para exponer los objetivos del estudio y solicitar su consentimiento para la realización 
del mismo. Luego de la autorización de la Institución para colaborar con la investigación, se solicitó el consentimiento informado por escrito a las familias. En un taller con las familias que se llevó a cabo en la clínica, se repartieron los cuestionarios que fueron auto administrados.

\section{Diseño}

Estudio no experimental de investigación empírica de tipo asociativo y transversal (Ato, López \& Benavente, 2013), con un muestreo de tipo no probabilístico, por conveniencia (Hernández, Fernández \& Baptista, 2010).

\section{Análisis de datos}

Los datos recogidos en este trabajo fueron analizados utilizando el programa SPSS Statistics 22. Para evaluar si las variables presentan una distribución normal, se utilizaron las pruebas Kolmogorov-Smirnov y la prueba de Levene. Se utilizó el procedimiento de contraste de hipótesis sobre una media, para comparar los resultados de la muestra clínica con los datos poblacionales. Para extraer la media poblacional, se utilizó las medias de una muestra normativa de 996 sujetos mayores de 12 años que contestaron el cuestionario FACES IV en Uruguay (Costa Ball, et al., 2009) y no diferían significativamente con las edades de los adolescentes de población normativa $(t=0,93 ; g l .=15 ; p<.36)$. En primer lugar, se compararon los puntajes medios en las escalas balanceadas (cohesión y flexibilidad); desbalanceadas (desligada, enmarañada, rígida y caótica) de las dimensiones cohesión y flexibilidad; en segundo lugar, se comparó los puntajes medios en las escalas de comunicación y satisfacción familiar entre los grupos de familias con un miembro con TCA y los datos de población normativa obtenidos en Uruguay (Costa Ball et al., 2009). Estos análisis se realizaron segmentando la base de datos por miembro de la familia utilizando el procedimiento prueba $t$ para una muestra, con el objetivo de contrastar hipótesis referidas a una media poblacional (Pardo \& Ruiz Diaz, 2002).

Se utilizó el programa $G *$ Power: Statistical Power Analyses for Windows and Mac, para analizar el tamaño del efecto de los resultados de las pruebas t para una muestra, este procedimiento nos cuantifica la diferencia entre dos medias (Faul, Erdfelder, Buchner, \& Lang, 2009). Para la valoración de la magnitud del tamaño del efecto se utilizó las recomendaciones de Cohen: valores próximos $\mathrm{a} d=.20$ efecto pequeño, $d=.50$ efecto moderado y $d$ $=.80$ efectos grandes (Cohen, 1988).

\section{Resultados}

En la Tabla 1 se muestran las medias y desvíos estándar de los 63 participantes, miembros de 16 familias con un miembro adolescente con TCA. En primer lugar, se utilizó la prueba Kolmogorov-Smirnov, para evaluar si las variables presentan una distribución normal. Los resultados encontrados con la $Z$ de Kolmogorov-Smirnov en todos las variables del FACES IV, presentaron un nivel crítico mayor a $p>$.05. Debiendo aceptar la hipótesis nula de normalidad, concluyendo que cada una de las ocho variables del FACES IV se ajusta a una distribución normal.

Tabla 1

Estadísticos descriptivos y análisis de normalidad de las escalas del FACES IV

\begin{tabular}{lccc}
\hline FACES IV & $\boldsymbol{M}$ & $\boldsymbol{D E}$ & Normalidad \\
\hline Cohesión & 16,05 & 2,95 & 1.21 \\
Flexibilidad & 13,49 & 3,09 & 0.66 \\
Desligada & 9,05 & 3,75 & 1.24 \\
Enmarañada & 9,75 & 2,80 & 0.80 \\
Rígida & 10,41 & 3,12 & 1.03 \\
Caótica & 10,03 & 4,17 & 0.89 \\
Comunicación & 35,35 & 7,30 & 1,01 \\
Satisfacción & 35,06 & 7,83 & 0.70 \\
\hline * p<.05 (Éstūdio de normalídad, usando Prueba de Kolmogorov-Smirnov \\
para una muestra) \\
* p<.05 (Estudio de homodedasticidad, usando el Estadístico de Levene \\
basándose en la medias)
\end{tabular}

En la Tabla 2 se muestran las medias, desvíos estándar y prueba de normalidad de todas las escalas del FACES IV, segmentada por la variable rol (paciente, hermano, padre y madre). Los resultados del estudio de normalidad (prueba Kolmogorov-Smirnov), en todos las variables del FACES IV, presentaron un nivel crítico mayor a $p>.05$. Debiendo aceptar la hipótesis nula de normalidad, concluyendo que todas las escalas del FACES IV se ajustan a una distribución normal cuando se las segmenta por rol. 
Tabla 2

Estadísticos descriptivos y análisis de normalidad del FACES IV por rol

\begin{tabular}{|c|c|c|c|c|c|c|c|c|c|c|c|c|}
\hline & \multicolumn{3}{|c|}{ Paciente } & \multicolumn{3}{|c|}{ Hermanos } & \multicolumn{3}{|c|}{ Madre } & \multicolumn{3}{|c|}{ Padre } \\
\hline & $M$ & $D E$ & $Z$ & $M$ & $D E$ & $Z$ & $M$ & $D E$ & $Z$ & $M$ & $D E$ & $Z$ \\
\hline Cohesión & 15.80 & 2.59 & 0.82 & 16.29 & 3.49 & 0.95 & 15.75 & 3.29 & 0.62 & 16.36 & 2.43 & 0.66 \\
\hline Flexibilidad & 11.44 & 2.55 & 0.59 & 12.53 & 2.76 & 0.50 & 14.33 & 2.44 & 0.80 & 15.93 & 2.76 & 0.71 \\
\hline Enmarañada & 9.56 & 2.03 & 0.79 & 9.94 & 2.77 & 0.78 & 9.31 & 3.09 & 0.67 & 10.21 & 3.42 & 0.68 \\
\hline Desligada & 9.67 & 4.08 & 0.54 & 8.35 & 2.69 & 0.82 & 9.60 & 4.08 & 0.53 & 8.62 & 4.35 & 0.89 \\
\hline Rigida & 10.25 & 1.84 & 0.56 & 9.71 & 3.65 & 0.52 & 9.88 & 2.70 & 1.01 & 12.33 & 3.75 & 0.59 \\
\hline Caótica & 11.44 & 3.79 & 0.45 & 10.47 & 4.33 & 0.52 & 9.63 & 4.33 & 0.66 & 8.23 & 3.94 & 0.58 \\
\hline Comunicación & 31.87 & 6.97 & 0.80 & 34.35 & 7.43 & 0.54 & 36.80 & 6.10 & 0.94 & 39.00 & 7.41 & 0.60 \\
\hline Satisfacción & 31.81 & 8.66 & 0.59 & 33.94 & 8.59 & 0.80 & 36.56 & 6.32 & 0.60 & 38.43 & 6.29 & 0.63 \\
\hline
\end{tabular}

${ }^{*} \mathrm{p}<.05$ (Estudio de normalidad, usando Prueba de Kolmogorov-Smirnov para una muestra)

En la Tabla 3, se muestran los análisis de la prueba T para una muestra para contrastar la hipótesis referida a una media poblacional. Se compararon las medias del total de participantes de la muestra clínica $(N=63)$ con las medias de la población normativa en todas las escalas del FACES IV publicadas en Costa Ball et al. (2009).

Según los datos presentados en la Tabla 3, la muestra clínica reporta puntajes significativamente descendidos en las escalas balanceadas (cohesión y flexibilidad), y en las escalas de comunicación y satisfacción familiar. Mientras que en las escalas desbalanceadas (desligada, enmarañada, rígida y caótica) se obtuvieron puntajes significativamente más altos, en comparación con la población normativa. En cinco escalas (cohesión, flexibilidad, desligada, comunicación y satisfacción) se encontraron que las magnitudes de los efectos oscilan en el entorno de 0.80 , significando un gran efecto. A su vez la escala Caótica presenta un efecto moderado y un efecto pequeño las escalas Enmarañadas y Rígida.

En la Tabla 4 se muestran los resultados de la prueba t para una muestra para contrastar la hipótesis referida a una media poblacional abriendo los resultados por la variable rol, junto a su respectiva valoración de la magnitud del tamaño de los efectos $(d)$.

Tabla 3

Prueba t para una muestra (diferencia entre población normativa y clínica) y valoración de la magnitud del tamaño del efecto (d)

\begin{tabular}{|c|c|c|c|c|c|c|}
\hline \multirow[b]{2}{*}{ Escalas del FACES IV } & \multicolumn{2}{|c|}{ Población normativa } & \multicolumn{2}{|c|}{ Muestra clínica } & \multirow[b]{2}{*}{$t$} & \multirow[b]{2}{*}{$d$} \\
\hline & $M$ & $D E$ & $M$ & $D E$ & & \\
\hline Cohesión & 18,19 & 2,11 & 16,05 & 2,956 & $-5,70^{* * *}$ & 0.73 \\
\hline Flexibilidad & 15,84 & 2,49 & 13,49 & 3,095 & $-6,02^{* * *}$ & 0.76 \\
\hline Desligada & 6,32 & 2,66 & 9,05 & 3,753 & $5,63^{\star \star \star *}$ & 0.73 \\
\hline Enmarañada & 10,62 & 3,35 & 9,75 & 2,805 & $-2,47^{\star}$ & 0.31 \\
\hline Rígida & 11,31 & 3,40 & 10,41 & 3,127 & $-2,24^{*}$ & 0.29 \\
\hline Caótica & 7,58 & 3,00 & 10,03 & 4,176 & $4,62^{\star \star \star}$ & 0.59 \\
\hline Comunicación & 41,28 & 5,95 & 35,35 & 7,309 & $-6,28^{\star \star \star}$ & 0.81 \\
\hline Satisfacción & 41,50 & 5,67 & 35,06 & 7,839 & $-6,51^{\star \star \star}$ & 0.82 \\
\hline
\end{tabular}

${ }^{*} p<.05 ;{ }^{* \star} p<.001 ;{ }^{* \star *} p<.000$ 
Tabla 4

Comparación de la percepción del funcionamiento de las FTCA respecto a las FN según rol

\begin{tabular}{|c|c|c|c|c|c|c|c|}
\hline \multirow[b]{2}{*}{ Escalas del FACES IV } & \multicolumn{2}{|c|}{ Población normativa } & \multicolumn{2}{|c|}{ Muestra clínica } & \multirow[b]{2}{*}{$T$} & \multirow[b]{2}{*}{$P$} & \multirow[b]{2}{*}{$d$} \\
\hline & $M$ & $D E$ & $M$ & $D E$ & & & \\
\hline \multicolumn{8}{|l|}{ Enmarañada } \\
\hline Pacientes & 10.42 & 2.86 & 9.56 & 2.03 & -1.68 & .112 & \\
\hline Hermanos & 10.42 & 2.86 & 9.94 & 2.77 & -0.72 & .478 & \\
\hline Madres & 10.32 & 3.52 & 9.31 & 3.09 & -1.30 & .212 & \\
\hline Padres & 10.88 & 3.33 & 10.21 & 3.42 & -.728 & .480 & \\
\hline \multicolumn{8}{|l|}{ Cohesión } \\
\hline Pacientes & 17.54 & 2.44 & 15,80 & 2.59 & -2.62 & $.02^{\star}$ & 0.67 \\
\hline Hermanos & 17.54 & 2.44 & 16.29 & 3.49 & -1.56 & .137 & \\
\hline Madres & 18.52 & 1.94 & 15.75 & 3.29 & -6.58 & $.000^{* * *}$ & 0.84 \\
\hline Padres & 18.29 & 2.05 & 16.36 & 2.43 & -2.96 & $.011^{\star \star}$ & 0.79 \\
\hline \multicolumn{8}{|l|}{ Desligada } \\
\hline Pacientes & 6.84 & 2.76 & 9.67 & 4.08 & 2.68 & $.018^{*}$ & 0.69 \\
\hline Hermanos & 6.84 & 2.76 & 8.35 & 2.69 & 2.42 & $.028^{\star}$ & 0.56 \\
\hline Madres & 6.01 & 2.58 & 9.60 & 4.08 & 3.40 & $.004^{* \star}$ & 0.88 \\
\hline Padres & 6.17 & 2.62 & 8.62 & 4.35 & 2.02 & .065 & \\
\hline \multicolumn{8}{|l|}{ Rígida } \\
\hline Pacientes & 11.06 & 3.45 & 10.25 & 1.84 & -1.75 & .099 & \\
\hline Hermanos & 11.06 & 3.45 & 9.75 & 3.27 & -.138 & .194 & \\
\hline Madres & 11.27 & 3.33 & 9.71 & 3.65 & -1.66 & .116 & \\
\hline Padres & 11.60 & 3.53 & 12.33 & 3.75 & 0.67 &, 512 & \\
\hline \multicolumn{8}{|l|}{ Flexibilidad } \\
\hline Pacientes & 14.83 & 2.68 & 11.44 & 2.55 & -5.31 & $.000^{\star \star \star}$ & 1.33 \\
\hline Hermanos & 14.83 & 2.68 & 12.53 & 2.76 & -3.65 & $.002^{\star \star}$ & 0.83 \\
\hline Madres & 16.11 & 2.17 & 14.33 & 2.44 & -2.82 & $.014^{*}$ & 0.73 \\
\hline Padres & 16.29 & 2.42 & 15.93 & 2.76 & -0.50 & .625 & \\
\hline \multicolumn{8}{|l|}{ Caótica } \\
\hline Pacientes & 7.82 & 2.78 & 11.44 & 3.61 & 3.81 & $.002^{\star \star}$ & 1.00 \\
\hline Hermanos & 7.82 & 2.78 & 10.47 & 4.33 & 2.65 & $.017^{\star}$ & 0.61 \\
\hline Madres & 7.38 & 3.24 & 9.63 & 4.33 & 2.07 & $.056^{*}$ & 0.52 \\
\hline Padres & 7.52 & 3.03 & 8.23 & 3.94 & 0.65 & .528 & 0.18 \\
\hline \multicolumn{8}{|l|}{ Comunicación } \\
\hline Pacientes & 38.65 & 6.77 & 31.87 & 6.97 & -3.76 & $.002^{\star \star}$ & 0.97 \\
\hline Hermanos & 38.65 & 6.77 & 34.35 & 7.43 & -.23 & .816 & \\
\hline Madres & 41.96 & 5.41 & 36.80 & 6.10 & -3.27 & $.006^{*}$ & 0.85 \\
\hline Padres & 42.51 & 5.19 & 39.00 & 7.41 & -1.70 & .114 & \\
\hline \multicolumn{8}{|l|}{ Satisfacción } \\
\hline Pacientes & 39.47 & 6.9 & 31.81 & 8.66 & -3.76 & $.002^{\star \star}$ & 0.88 \\
\hline Hermanos & 39.47 & 6.9 & 33.94 & 8.59 & -2.80 & $.013^{*}$ & 0.64 \\
\hline Madres & 41.93 & 5.29 & 36.56 & 6.32 & -3.39 & $.004^{\star \star}$ & 0.85 \\
\hline Padres & 42.46 & 5.15 & 38.43 & 6.29 & -2.39 & $.032^{\star}$ & 0.64 \\
\hline
\end{tabular}

A continuación analizaremos los resultados desagregando por rol (padre, madre, hermano y paciente), agrupándolos en: 1. escalas balanceadas (cohesión y flexibilidad), 2. desbalanceadas (enmarañada, desligada, rígida y caótica), 3. comunicación familiar y 4. satisfacción familiar.

1. En las escalas de cohesión y flexibilidad, familiar los resultados muestran que existen diferencias estadísticamente significativas entre la muestra clínica y la población normativa, en la mayoría de los contrastes de hipótesis sobre una media (siempre a favor de la población normativa). Las diferencias encontradas entre la muestra clínica y la población normativa, cuando se estudia la escala de cohesión, corresponden a magnitudes de efectos grandes en las diferencias encontradas entre madres y padres respecto a la población normativa, y moderados con la paciente en la escala de cohesión. Mientras en la escala de flexibilidad, se encontraron magnitudes de efectos grandes en las diferencias estadísticamente significativas encontradas entre pacientes, hermanos y madres, cuando se la comparan con población normativa.

2. En las escalas desbalanceadas (enmarañada y desligada de la dimensión cohesión; rígida y caótica de la dimensión flexibilidad) desagregadas por rol, no existen diferencias estadísticamente significativas en las escalas rígida y enmarañada cuando se las compara con población normativa según rol (paciente, hermano, padre y madre). Mientras que en las restantes escalas (desligada y caótica) existen diferencias estadísticamente significativas en el rol paciente, hermano y madre, a favor de la muestra normativa. Mientras en la escala caótica, ocurre lo mismo. Las madres, hermanos y pacientes se perciben más desligados 
y más caóticos en la muestra clínica que en los datos de la población normativa. Únicamente se encontraron diferencias estadísticamente significativas correspondientes a magnitudes de efectos grandes entre madres y población normativa en la escala desligada y entre pacientes y población normativa en la escala caótica. En el resto de los contrastes las magnitudes de los efectos son moderados.

3. En la escala de comunicación, existen diferencias estadísticamente significativas a favor de la población normativa, entre las madres y pacientes. Mientras que no existen diferencias significativas entre padres y hermanos. Las diferencias encontradas corresponden a magnitudes de efectos grandes para los pacientes y madres, para los restantes (hermanos y padres) le corresponden magnitudes de efectos moderados.

4. En la escala de satisfacción familiar, existen diferencias estadísticamente significativas por rol (padre, madre, hermanos y pacientes) a favor de la población normativa. Las diferencias encontradas corresponden a magnitudes de efectos grandes cuando se comparan la satisfacción familiar entre pacientes o hermanos, frente a los resultados de población normativa. El resto de los contrastes corresponden a magnitudes de efecto moderados.

\section{Conclusiones}

El primer objetivo de este trabajo fue describir el funcionamiento de las familias con un miembro adolescente con TCA en una pequeña muestra uruguaya según el Modelo Circumplejo. Se administró el instrumento FACES-IV versión uruguaya porque es el instrumento del Modelo Circumplejo, y por ser considerado uno de los métodos de evaluación familiar más utilizados para evaluar su funcionamiento.

Respecto a nuestro segundo objetivo, comparar el funcionamiento familiar entre familias con un miembro adolescente con TCA y las Familias sin Trastorno, podemos afirmar que las familias estudiadas poseen menor cohesión. Esta característica observada se alinea con los resultados obtenidos en los estudios de (Dancyger et al., 2005; Manglio \& Molina, 2012; Vázquez, et al., 2010). En la dimensión flexibilidad, hay estudios que coinciden con nuestros resultados (Vidovic et al., 2005; Waller et al., 1990) en cuanto a que las familias con TCA poseen menor flexibilidad, mientras que otros investigadores no encuentran diferencias significativas entre estas familias y las familias control (Cook-Darezens et al., 2005; Manglio \& Molina, 2012).

En relación a las escalas desbalanceadas, las familias con TCA muestran un funcionamiento caótico, desligado, enmarañado y rígido en comparación con las familias normativas. En la muestra clínica se observan familias de tipo desbalanceadas, dado que presentaron niveles bajos de cohesión y flexibilidad y altos en las escalas: desligada, caótica, enmarañada y rígida; mientras que las familias de la población normativa se caracterizan por puntajes altos en las escalas balanceadas y bajos en las escalas desbalanceadas. Por otra parte, estos resultados concuerdan con la hipótesis de Olson (2003): puntajes bajos en las escalas balanceadas y altos en las escalas desbalanceadas, estarían asociados a un funcionamiento problemático. A su vez, coinciden con los estudios que afirman que estas familias tienden a ser más disfuncionales que las familias control (Cruzat et al., 2008; Holtom-Viesel \& Allan, 2014; Ruiz et al., 2013).

En lo referente a la tercera dimensión, en la escala de comunicación, las familias con TCA, obtienen puntajes descendidos en comparación con las familias normativas. Estos resultados se alinean con los postulados de (Crispo et al., 1996; Selvini-Palazzoli et al., 1999). También coinciden con una de las hipótesis básicas del modelo de Olson y Gorall (2003), respecto a que sistemas desbalanceados en estas dos dimensiones tienden a una pobre comunicación. El síntoma parecería ser una de las expresiones de ésta.

Vale destacar que desagregando los datos por rol, es decir por integrante de la familia, las madres y las pacientes (TCA) coinciden con la percepción del funcionamiento familiar. Ambas reportan un funcionamiento familiar idéntico, descendido en las escalas balanceadas (cohesión y flexibilidad). Mientras que en las escalas desbalanceadas, madres y pacientes refieren un funcionamiento desligado y caótico, con una pobre comunicación y satisfacción familiar que se acerca a la percepción de los hermanos. Por otra parte, los padres de la muestra perciben una mayor flexibilidad y en las escalas caótica y desligada, no reportan disfuncionalidad. Esto 
podría vincularse con una ubicación periférica del rol paterno en el sistema. Llama la atención, sin embargo, que padre y madre coinciden en reportar una baja cohesión.

A nuestro entender, estas coincidencias madre - hija - hermanos y discrepancias del padre con el resto del sistema, salvo en la escala de cohesión, dan cuenta de las marcadas diferencias de las familias TCA con las familias del grupo control y confirman la necesidad de profundos cambios en el sistema. Resulta significativo también, el hecho de que todos los miembros coincidan en reportar baja satisfacción familiar.

En familias no clínicas cursando la etapa con hijos adolescentes, se espera que los hijos muestren discrepancias con sus madres y padres. En las familias estudiadas, la total coincidencia de la percepción de las madres y sus hijas, podrían estar dando cuenta de una inadecuada diferenciación con respecto a lo esperado según la etapa evolutiva familiar que están transitando.

Este estudio confirma en una pequeña muestra local, lo descubierto en investigaciones anteriores y de otras latitudes, presentando grandes desafíos para la intervención en los TCA. Una vez más, se plantea a los clínicos la necesidad de trabajar con todo el sistema familiar, para abordar la problemática de los TCA de las adolescentes. Las disonancias entre los distintos miembros del sistema, están indicando un camino en cuanto al trabajo con la proximidad y distancia de los miembros de la familia. Holtom-Viesel y Allan (2014) también señalan que la intervención en el funcionamiento familiar puede convertirse en un recurso clave para mejorar la percepción del paciente en cuanto al sistema y así facilitar su recuperación.

Este trabajo pretende ser un pequeño aporte tanto a la estandarización de la técnica en la versión uruguaya 2012, como a la clínica para la comprensión de las familias con TCA en el país.

\section{Referencias}

American Psychiatric Association. (2013). The Diagnostic and Statistical Manual of Mental Disorders (5a ed.). Washington, DC.: American Psychiatric Publishing.

Ato, M., López, J. \& Benavente, A. (2013). Un sistema de clasificación de los diseños de investigación en psicología. Anales de psicología, 29(3), 1038-1059.

Cohen, J. (1988). Statistical power analysis for the behavioral sciences. New Jersey: Lawrence Erlbaum Associates.
Cook-Darzens, S., Doyen, C., Falissard, B., \& Mouren, M.C. (2005). Self- perceived family functioning in 40 French families of anorexic adolescents: implications for therapy. European Eating Disorders Review, 13, 223-226.

Correa, M. L., Zubarew, T., Silva, P., \& Romero, M. (2006). Prevalencia de riesgo de trastornos alimentarios en adolescentes mujeres escolares de la Región Metropolitana. Revista Chilena de Pediatría, 77(2), 153-160.

Costa-Ball, D., González Tornaría, M., del Arca, D., Masjuan, N., \& Olson, D.H. (2013). Adaptación al Uruguay del Cuestionario FACES IV: Propiedades psicométricas. Ciencias Psicológicas, 7(2), 119-132.

Costa-Ball, D., González Tornaría, M., Masjuan, N., Trápaga, M., Del Arca, D., Scafarelli, L., \& Feibuscheurez, A. (2009). Escala de evaluación del funcionamiento familiar -FACES IV-: proceso de adaptación a Montevideo, Uruguay. Ciencias Psicológicas, 3(1), 43-56.

Cruzat, C., Ramírez, P., Melipillán, R., \& Marzolo, P. (2008). Trastornos Alimentarios y Funcionamiento Familiar Percibido en una Muestra de Estudiantes Secundarias de la Comuna de Concepción. Psykhe, 17(1), 81-90.

Dancyger, I., Fornari, V., Scionti, L., Wistsky, W., \& Sunday, S. (2005). Do daughters with eating disorders agree with their parents perception of Family Functioning? Comprehensive Psychiatry, 46, 135-139.

Espina, A., (1996). Terapia Familiar en la Anorexia Nerviosa. El Modelo Sistémico. En A. Espina y B. Pumar (Eds.), Terapia Familiar Sistémica. Teoría, Clínica e Investigación, (pp. 154-183). Madrid: Fundamentos.

Faul, F., Erdfelder, E., Buchner, A. \& Lang, A. G. (2009). Statistical power analyses using G*Power 3.1: Tests for correlation and regression analyses. Behavior Research Methods, 41(4), 1149-1160.

Feibuscheurez, A. (2009). Características del funcionamiento familiar en el que un miembro padece un trastorno de conducta alimentaria. Manuscrito inédito. Montevideo: Facultad de Psicología.

Crispo, R., Figueroa, E., \& Guelar, D. (1996) Anorexia y Bulimia. Lo que hay que saber. Buenos Aires: Paidós.

Hernández, R., Fernández, C. \& Baptista, P. (2010). Metodología de la investigación (5a ed.). México: Mc Graw Hill.

Holtom-Viesel, A., \& Allan, S. (2014). A systematic review of the literature on family functioning across all eating disorder diagnoses in comparison to control families. Clinical Psychology Review, 34, 29-43.

IBM Corporation (2012a). IBM SPSS Statistics (22). Recuperado de http://www-01.ibm.com/software/ es/ analytics/spss/

Kouneski, E.F. (2000). The Family Circumplex Model, FACES II, and FACES IIII: Overview of Research and pplications. Recuperado de http://www.facesiv.com/ pdf/faces_and_circumplex.pdf

Manglio, A.L., \& Molina, M.F. (2012). La familia de adolescentes con trastornos alimentarios ¿Cómo perciben sus miembros el funcionamiento familiar? Revista Mexicana de Trastornos Alimentarios, 3, 1-10.

March, J.C., Suess, A., Prieto, M.A., Escudero, M., Nebot, M., Cabeza, E., \& Pallicer, A. (2006). Trastornos de la Conducta Alimentaria: Opiniones y expectativas sobre estrategias de prevención y tratamiento desde la perspectiva de diferentes actores sociales. Nutr Hosp, 21(1), 4-12. 
Montero, L., \& León, O. (2002). Clasificación y descripción de las metodologías de investigación en psicología. International Journal of Clinical and Health Psychology, 2(3), 503-508.

Olson, D.H. (2000). Circumplex Model of Marital and Family Systems. Journal of Family Therapy, 22, 144-167.

Olson, D.H. (2011). FACES IV and the Circumplex Model: Validation Study. Journal of Marital \& Family Therapy, 3(1), 64-80.

Olson, D.H., \& Gorall, D.M. (2003). Circumplex model of marital and family systems. En F. Walsh, Normal Family Processes (3a ed.) (pp. 514-547). New York: Guilford.

Olson, D.H., Sprenkle, D.H., \& Russell, C.S. (1979). Circumplex Model of Marital and Family Systems: I. Cohesion and Adaptability Dimensions, Family Types, and Clinical Applications. Family Process, 18(1), 3-28.

Pardo, A., \& Ruiz Diaz, M.A. (2002). SPSS 11. Guía para el análisis de datos. Madrid: Mc Graw Hill.

Ruiz, A., Vázquez, R., Mancilla, J., Viladrich, C., \& Castillo, H. (2013). Factores Familiares asociados a los Trastornos Alimentarios: una revisión. Revista Mexicana de Trastornos Alimentarios, 4, 45-57.
Sanz Vázquez, M. (2008a). Escala de Comunicación Familiar. En Equipo EIF, Manual de instrumento de evaluación familiar, (pp. 79-87). Madrid: Ed. CCS.

Sanz Vázquez, M. (2008b). Escala de Satisfacción Familiar. En Equipo EIF, Manual de instrumento de evaluación familiar, (pp. 69-77). Madrid: Ed. CCS.

Selvini-Palazzoli, M., Cirillo, M., Selvini, M.S., \& Sorrentino, A.M. (1999). Muchachas anoréxicas y bulímicas. Buenos Aires: Paidos.

Vázquez, R., Ruíz, A., Álvarez, G., Mancilla, J., \& Tena, A. (2010). Percepción del funcionamiento familiar de mujeres con trastornos del comportamiento alimentario. Psicología Conductual, 18(1), 105-117.

Vidovic, V., Jures, V., Begovac, I., Mahnik, M., \& Tocilj, G. (2005). Perceived family cohesion, adaptability, and communication in eating disorders. European Eating Disorders Review, 13, 19-28.

Para citar este artículo:

Fernández Ruiz, M., Masjuan, N., Costa-Ball, D., \& Cracco, C. (2015). Funcionamiento Familiar y Trastornos de la Conducta Alimentaria. Una investigación desde el Modelo Circumplejo.

Ciencias Psicológicas 9(2): 153 - 162 